Research Article

\title{
Reliability Sensitivity Analysis Method for Mechanical Components
}

\author{
Yan-Fang Zhang ${ }^{1}{ }^{1}$ and Yan-Lin Zhang $\mathbb{D}^{2}$ \\ ${ }^{1}$ Department of Basic Course, Institute of Disaster Prevention, Sanhe 065201, China \\ ${ }^{2}$ North China Institute of Science \& Technology, Sanhe 065201, China \\ Correspondence should be addressed to Yan-Fang Zhang; zyf@cidp.edu.cn
}

Received 21 April 2021; Revised 19 June 2021; Accepted 6 July 2021; Published 16 July 2021

Academic Editor: Arturo J. Fernández

Copyright (c) 2021 Yan-Fang Zhang and Yan-Lin Zhang. This is an open access article distributed under the Creative Commons Attribution License, which permits unrestricted use, distribution, and reproduction in any medium, provided the original work is properly cited.

\begin{abstract}
Based on the univariate dimension-reduction method (UDRM), Edgeworth series, and sensitivity analysis, a new method for reliability sensitivity analysis of mechanical components is proposed. The univariate dimension-reduction method is applied to calculate the response origin moments and their sensitivity with respect to distribution parameters (e.g., mean and standard deviation) of fundamental input random variables. Edgeworth series is used to estimate failure probability of mechanical components by using first few response central moments. The analytic formula of reliability sensitivity can be derived by calculating partial derivative of the failure probability $P_{\mathrm{f}}$ with respect to distribution parameters of basic random variables. The nonnormal random parameters need not to be transformed into equivalent normal ones. Three numerical examples are employed to illustrate the accuracy and efficiency of the proposed method by comparing the failure probability and reliability sensitivity results obtained by the proposed method with those obtained by Monte Carlo simulation (MCS).
\end{abstract}

\section{Introduction}

The responses of mechanical components or engineering structures are often random due to random inputs of them including loads, material properties, and geometry. Many reliability analysis methods have been developed to calculate the failure probabilities of these random structures [1-4]. In reliability analysis, reliability sensitivity is defined as the partial derivative of the failure probability with respect to the distribution parameters (e.g., mean and standard deviation) of fundamental random input variables. Reliability sensitivity provides information about the importance of each input random variable to a structure's failure probability. Reliability sensitivity analysis methods can be divided into the numerical simulation method and approximate analytic calculation method.

The numerical simulation method can be divided into many different methods due to different sampling methods including importance sampling, direction sampling, line sampling, subset simulation, and low-discrepancy sampling
[5-10]. These different simulation methods are all based on Monte Carlo simulation but have different sampling methods. Although the program of these methods is not complicated, lots of samples are needed to get precise results because the structural failure probability is often very small and the computational efficiency is therefore reduced.

Analytic methods for reliability sensitivity analysis are always based on analytic reliability methods. These reliability analysis methods are the mean-value first-order reliability method (MVFORM)/mean-value second-order reliability method (MVSORM) [11, 12], JC method, mean-value firstorder saddlepoint approximation (MVFOSA) [13], and moment method. FORM/SORM needs to expand the performance function into first-order or second-order Taylor series at the most likely failure point. The expansion is widely used because of its relatively moderate precision and efficiency. The corresponding reliability sensitivity calculation methods have been derived from these reliability methods [14-23]. Analytic methods for reliability sensitivity analysis always have higher calculation efficiency than MCS. 
In this study, a feasible method to compute the sensitivity of failure probability with respect to distribution parameters of basic random input variables is proposed. The method can be applied to estimate failure probability and reliability sensitivity based on Edgeworth series. The proposed reliability sensitivity analysis method includes the following steps. (1) Choose random input variables and determine their distributions and distribution parameters. (2) Calculate the first four response origin moments and their sensitivity with respect to distribution parameters of random input variables by UDRM. (3) Calculate failure probability by the reliability method based on Edgeworth series. (4) Calculate partial derivatives of failure probability with respect to response central moments. (5) Calculate partial derivatives of response central moments with respect to response origin moments. (6) Calculate reliability sensitivity defined by partial derivatives of failure probability with respect to distribution parameters of input random variables.

\section{Response Origin Moments and Response Sensitivity}

2.1. Response Origin Moments by UDRM. Structures subject to random input vector $\mathbf{X}=\left[X_{1}, X_{2}, \Lambda, X_{N}\right]^{T} \in R^{N}$, which characterizes uncertainty in loads, material properties, and geometry. Let $Y(\mathbf{X})$ represent a response of interest that depends on independent random variables $\mathbf{X}=\left[X_{1}\right.$, $\left.X_{2}, \Lambda, X_{N}\right]^{T}$; then, the $l^{\text {th }}$ order origin moment of $Y(\mathbf{X})$ can be written as follows:

$$
m_{l}=E\left[Y^{l}(\mathbf{X})\right]=\int_{\mathrm{R}^{N}} Y^{l}(\mathbf{X}) f_{\mathbf{X}}(\mathbf{x}) \mathrm{d} \mathbf{x}
$$

where $m_{l}$ is the $l^{\text {th }}$ order origin moment of $Y(\mathbf{X}), f_{\mathbf{X}}(\mathbf{x})$ is the joint probability density function of $\mathbf{X}$, and $E(\cdot)$ is the expectation operator. According to the univariate dimension reduction method proposed by Rahman and $\mathrm{Xu}$ [24-26], $Y(\mathbf{X})$ can be approximately written as follows:

$$
Y(\mathbf{X}) \approx \hat{Y}(\mathbf{X})=\widehat{Y}\left(X_{1}, X_{2}, \Lambda, X_{N}\right)=\sum_{j=1}^{N} Y\left(\mu_{1}, \Lambda, \mu_{j-1}, x_{j}, \mu_{j+1}, \Lambda, \mu_{N}\right)-(N-1) Y\left(\mu_{1}, \Lambda, \mu_{N}\right)
$$

where $Y\left(\mu_{1}, \Lambda, \mu_{j-1}, x_{j}, \mu_{j+1}, \Lambda \mu_{N}\right)$ is the response which depends on the $j^{\text {th }}$ random input $X_{j}$, $Y\left(\mu_{1}, \Lambda, \mu_{j-1}, x_{j}, \mu_{j+1}, \Lambda \mu_{N}\right)$ is the response where $\mathbf{X}=\left[X_{1}, X_{2}, \Lambda, X_{N}\right]^{T}$, and $\mu_{j}=E\left(X_{j}\right)$ is the mean of $X_{j}$.
Then, the $l^{\text {th }}$ order origin moment $m_{l}$ of $Y(\mathbf{X})$ can be written as follows:

$$
m_{l} \cong E\left[\widehat{Y}^{l}(\mathbf{X})\right]=E\left\{\left[\sum_{j=1}^{N} Y\left(\mu_{1}, \Lambda, \mu_{j-1}, X_{j}, \mu_{j+1}, \Lambda, \mu_{N}\right)-(N-1) Y\left(\mu_{1}, \Lambda, \mu_{N}\right)\right]^{l}\right\}
$$

Applying the binomial formula on the right-hand side of equation (3), the $l^{\text {th }}$ response origin moment $m_{l}$ can be written as follows:

$$
m_{l} \cong \sum_{i=0}^{l} \mathrm{C}_{l}^{i} E\left[\sum_{j=1}^{N} Y\left(\mu_{1}, \Lambda, \mu_{j-1}, X_{j}, \mu_{j+1}, \Lambda, \mu_{N}\right)\right]^{i}\left[-(N-1) Y\left(\mu_{1}, \Lambda, \mu_{N}\right)\right]^{l-i},
$$

where $C(\cdot)$ is the combination operator.

Define

$$
S_{j}^{i}=E\left\{\left[\sum_{i=1}^{j} Y\left(\mu_{1}, \Lambda, \mu_{j-1}, X_{j}, \mu_{j+1}, \Lambda, \mu_{N}\right)\right]^{i}\right\}, \quad j=1, \ldots N, i=1, \ldots, l .
$$

Equation (5) can be expressed using the recursive formula as follows [23]: 


$$
\begin{aligned}
& S_{1}^{i}=E\left[Y^{i}\left(X_{1}, \mu_{2}, \Lambda, \mu_{N}\right)\right], \quad(i=1, \ldots, N), \\
& S_{2}^{i}=\sum_{k=0}^{i} C_{i}^{k} S_{1}^{i} E\left[Y^{i-k}\left(\mu_{1}, X_{2}, \Lambda, \mu_{N}\right)\right], \quad(i=1, \ldots, N), \\
& S_{j}^{i}=\sum_{k=0}^{i} C_{i}^{k} S_{j-1}^{i} E\left[Y^{i-k}\left(\mu_{1}, \Lambda, \mu_{j-1}, X_{j}, \mu_{j+1}, \Lambda, \mu_{N}\right)\right], \quad(i=1, \ldots, N), \\
& S_{N}^{i}=\sum_{k=0}^{i} C_{i}^{k} S_{N-1}^{i} E\left[Y^{i-k}\left(\mu_{1}, \Lambda, \mu_{N-1}, X_{N}\right)\right], \quad(i=1, \ldots, N) .
\end{aligned}
$$
follows:

Then, the $l^{\text {th }}$ origin moment of $Y(\mathbf{X})$ can be written as

$$
m_{l} \cong E\left[\widehat{Y}^{l}(\mathbf{X})\right]=\sum_{i=0}^{l} C_{l}^{i} S_{N}^{i}\left[-(N-1) Y\left(\mu_{1}, \Lambda, \mu_{N-1}, \mu_{N}\right)\right]^{l-i}
$$

From equations (6) and (7), the $l^{\text {th }}$ response origin moment $m_{l}$ can be calculated out by using the origin moments of univariate functions $Y\left(\mu_{1}, \Lambda, \mu_{j-1}, X_{j}, \mu_{j+1}\right.$, $\left.\Lambda, \mu_{N}\right)(j=1,2, \Lambda, N)$. The $q^{\text {th }}$ origin moment of univariate function $Y\left(\mu_{1}, \Lambda, \mu_{j-1}, X_{j}, \mu_{j+1}, \Lambda, \mu_{N}\right)(j=1,2, \Lambda, N)$ can be written as follows:

$$
m_{Y_{j}}^{q}=E\left[Y^{q}\left(\mu_{1}, \Lambda, \mu_{j-1}, X_{j}, \mu_{j+1}, \Lambda, \mu_{N}\right)\right]=\int Y^{q}\left(\mu_{1}, \Lambda, \mu_{j-1}, x_{j}, \mu_{j+1}, \Lambda, \mu_{N}\right) f_{X_{j}}\left(x_{j}\right) \mathrm{d} x_{j}
$$

where $f_{X_{j}}\left(x_{j}\right)$ is the probability density function of random variable $X_{j}$.
The $q^{\text {th }}$ origin moment $m_{Y}^{q}$ in equation (8) can be calculated out by the numerical integration method and can be written as follows:

$$
m_{Y_{j}}^{q}=\int Y^{q}\left(\mu_{1}, \Lambda, \mu_{j-1}, x_{j}, \mu_{j+1}, \Lambda, \mu_{N}\right) f\left(x_{j}\right) \mathrm{d} x_{j}=\sum_{k=0}^{n} W_{j}^{(k)} Y^{q}\left(\mu_{1}, \Lambda, \mu_{j-1}, x_{j}^{(k)}, \mu_{j+1}, \Lambda, \mu_{N}\right),
$$

where $W_{j}^{(k)}$ is the $k^{\text {th }}$ integral weight, $x_{j}^{(k)}$ is the $k^{\text {th }}$ integration point, and $n$ is the number of integration points.

If $x_{j}$ has different probability density (e.g., normal, lognormal, and Weibull), the corresponding integral weights and integration points in equation (9) are different. The numerical integration points and the corresponding orthogonal polynomial for different probability densities are listed in Table 1 [27].
2.2. Sensitivity of Response Moment with Respect to Distribution Parameters of Inputs. Calculate the partial derivative on both side of equation (1) with respect to distribution parameters $T_{j}^{i}$ of random input variables $\mathbf{X}=\left[X_{1}, X_{2}, \Lambda, X_{N}\right]^{T}$. The partial derivative can be written as follows:

$$
\begin{aligned}
\frac{\partial m_{l}}{\partial T_{j}^{i}} & =\frac{\partial E\left(Y^{l}(\mathbf{X})\right)}{\partial T_{j}^{i}}=\frac{\partial\left(\int_{R^{N}} Y^{l}(\mathbf{X}) f(X) \mathrm{d} x\right)}{\partial T_{j}^{i}}=\int_{R^{N}} Y^{l}(\mathbf{X}) \frac{1}{f_{j}(x)} \cdot \frac{\partial f_{j}(x)}{\partial T_{j}^{i}} f(\mathbf{X}) \mathrm{d} x \\
& =E\left(Y^{l}(\mathbf{X}) \frac{1}{f_{j}(x)} \cdot \frac{\partial f_{j}(x)}{\partial T_{j}^{i}}\right) \\
& \approx E\left\{\left[\sum_{j=1}^{N} \widehat{Y}_{j}\left(\mathbf{X}_{j}\right)-(N-1) Y\left(\mu_{1}, \Lambda, \mu_{N}\right)\right]^{l} \frac{1}{f_{j}(x)} \cdot \frac{\partial f_{j}(x)}{\partial T_{j}^{i}}\right\}, \quad j=1,2, \ldots, N, i=1,2,
\end{aligned}
$$


TABLE 1: Orthogonal polynomial and Gauss integration.

\begin{tabular}{lcc}
\hline Distribution & Orthogonal polynomial & Integral formula \\
\hline Normal & Hermite & $(1 / \sqrt{\pi}) \sum_{k=0}^{n} W_{j}^{(k)} Y^{q}\left(\sqrt{2} \sigma t_{j}^{(k)}+\mu\right)$ \\
Lognormal & Hermite & $(1 / \sqrt{\pi}) \sum_{k=0}^{n} W_{j}^{(k)} Y^{q}\left(e^{\sqrt{2}} \sigma_{1} t_{j}^{(k)}+\mu_{1}\right)$ \\
Uniform & Legendre & $(1 / 2) \sum_{k=0}^{n} W_{l}^{(k)} Y^{q}\left((b-a / 2) t_{j}^{(k)}+(a+b / 2)\right)$ \\
Exponential & Laguerre & $(1 / \lambda) \sum_{k=0}^{n} W_{j}^{(k)} Y^{q}\left(t_{j}^{(k)} / \lambda\right)$ \\
Weibull & Laguerre & $\sum_{k=0}^{n} W_{j}^{(k)} Y^{q}\left(\alpha t_{j}^{1 / \beta}\right)$ \\
\hline
\end{tabular}

where $T_{j}^{i}(j=1,2, \ldots, N, i=1,2)$, is the $i^{\text {th }}$ distribution parameter of the $j^{\text {th }}$ random input variable $(i=1$, represents the mean; $i=2$ represents standard deviation), $f_{j}(x)$ is the probability density function of the $j^{\text {th }}$ random variable $X_{j}$, and $\widehat{Y}_{j}\left(\mathbf{X}_{j}\right)=Y\left(\mu_{1}, \Lambda, \mu_{j-1}, X_{j}, \mu_{j+1}, \Lambda, \mu_{N}\right)$.

Applying the binomial formula on the right-hand side of equation (10), $\partial m_{l} / \partial T_{j}^{i}$ can be written as follows:

$$
\frac{\partial m_{l}}{\partial T_{j}^{i}} \approx \sum_{z=0}^{l} C_{l}^{z} E\left\{\left[\sum_{j=1}^{N} \widehat{Y}_{j}\left(\mathbf{X}_{j}\right)\right]^{z} \frac{1}{f_{j}(x)} \cdot \frac{\partial f_{j}(x)}{\partial T_{j}^{i}}\right\}\left[-(N-1) Y\left(\mu_{1}, \Lambda, \mu_{N}\right)\right]^{l-z}
$$

where $\partial m_{l} / \partial T_{j}^{i}$ can be calculated out by equation (11) and recursive formula similar to equations (5) and (6).

Suppose

$$
k_{j}^{i}=\frac{1}{f_{j}(x)} \cdot \frac{\partial f_{j}(x)}{\partial T_{j}^{i}}, \quad j=1,2, \ldots, N, i=1,2,
$$

where $k_{j}^{i}$ is the kernel function of the $i^{\text {th }}$ distribution parameter $T_{j}^{i}$ of $j^{\text {th }}$ the random input variable.

The kernel function in equation (12) of $X_{j}$ with different probability density functions can be obtained as follows: here, $x_{j}$ is denoted by $x$.

If $X$ is a normal distributed variable, its probability density function can be written as follows:

$$
f(x)=\frac{1}{\sqrt{2 \pi} \sigma} e^{-\left((x-\mu)^{2} / 2 \sigma^{2}\right)},
$$

where $\mu$ is the mean of $X$, and $\sigma$ is the standard deviation of $X$.

The kernel function for normal variable $X$ with respect to its distribution parameters (mean and standard deviation) can be derived from equation (12) directly and can be written as follows:

$$
\begin{aligned}
& k_{\mu}=\frac{x-\mu}{\sigma^{2}} \\
& k_{\sigma}=\frac{1}{\sigma}\left(\left(\frac{x-\mu}{\sigma}\right)^{2}-1\right),
\end{aligned}
$$

where $k_{\mu}$ and $k_{\sigma}$ are the kernel functions with respect to mean and standard deviation, respectively. $\mu$ and $\sigma$ are the mean and standard deviation of normal variable $X$, respectively.
If $X$ is a lognormal distributed variable, its probability density function can be written as follows:

$$
f(x)=\frac{1}{\sqrt{2 \pi} \sigma_{1} x} e^{-\left(\left(\ln x-\mu_{1}\right)^{2} / 2 \sigma_{1}^{2}\right)},
$$

where $\mu_{1}$ is the mean of $\ln X$, and $\sigma_{1}$ is the standard deviation of $\ln X$.

The kernel function of lognormal variable $X$ with respect to $\mu_{1}$ and $\sigma_{1}$ can be derived from equation (12) directly and can be written as follows:

$$
\begin{aligned}
& k_{\mu_{1}}=\frac{\ln (x)-\mu_{1}}{\sigma_{1}^{2}}, \\
& k_{\sigma_{1}}=\frac{\left[\ln (x)-\mu_{1}\right]^{2}-\sigma_{1}^{2}}{\sigma_{1}^{3}},
\end{aligned}
$$

where $k_{\mu_{1}}$ and $k_{\sigma_{1}}$ are the kernel functions with respect to $\mu_{1}$ and $\sigma_{1}$, respectively.

Distribution parameters $\mu_{1}$ and $\sigma_{1}$ of the lognormal distributed variable $x$ can be written as follows:

$$
\begin{gathered}
\mu_{1}=\ln \left(\frac{\mu^{2}}{\sqrt{\sigma^{2}+\mu^{2}}}\right), \\
\sigma_{1}=\sqrt{\ln \left(\frac{\sigma^{2}}{\mu^{2}+1}\right)},
\end{gathered}
$$

where $\mu$ and $\sigma$ are the mean and standard deviation of the lognormal distributed variable $x$, respectively.

Calculate partial derivative of $\mu_{1}$ and $\sigma_{1}$ with respect to $\mu$ and $\sigma$, respectively, according to equation (16); then, 


$$
\begin{aligned}
& \frac{\partial \mu_{1}}{\partial \mu}=\frac{2 \sigma^{2}+\mu^{2}}{\mu\left(\sigma^{2}+\mu^{2}\right)}, \\
& \frac{\partial \mu_{1}}{\partial \sigma}=-\frac{2 \sigma}{\sigma^{2}+\mu^{2}}, \\
& \frac{\partial \sigma_{1}}{\partial \mu}=\frac{\mu}{\sqrt{\ln \left(\sigma^{2} / \mu^{2}+1\right)} \cdot\left(\mu^{2}+1\right)}, \\
& \frac{\partial \sigma_{1}}{\partial \sigma}=\frac{1}{\sigma \sqrt{\ln \left(\sigma^{2} / \mu^{2}+1\right)}} .
\end{aligned}
$$

Jacobi matrix $\mathbf{J}$ can be written as follows:

$$
\mathbf{J}=\left[\begin{array}{ll}
\frac{\partial \mu_{1}}{\partial \mu} & \frac{\partial \sigma_{1}}{\partial \mu} \\
\frac{\partial \mu_{1}}{\partial \sigma} & \frac{\partial \sigma_{1}}{\partial \sigma}
\end{array}\right]
$$

The kernel function of the lognormal distributed variable $X$ with respect to its mean and standard deviation can be written as follows:

$$
\left[\begin{array}{l}
k_{\mu} \\
k_{\sigma}
\end{array}\right]=\left[\begin{array}{l}
\frac{\partial \mu_{1}}{\partial \mu} k_{\mu_{1}}+\frac{\partial \sigma_{1}}{\partial \mu} k_{\sigma_{1}} \\
\frac{\partial \mu_{1}}{\partial \sigma} k_{\mu_{1}}+\frac{\partial \sigma_{1}}{\partial \sigma} k_{\sigma_{1}}
\end{array}\right]=\left[\begin{array}{ll}
\frac{\partial \mu_{1}}{\partial \mu} & \frac{\partial \sigma_{1}}{\partial \mu} \\
\frac{\partial \mu_{1}}{\partial \sigma} & \frac{\partial \sigma_{1}}{\partial \sigma}
\end{array}\right] \cdot\left[\begin{array}{l}
k_{\mu_{1}} \\
k_{\sigma_{1}}
\end{array}\right]=\mathbf{J} \cdot\left[\begin{array}{l}
k_{\mu_{1}} \\
k_{\sigma_{1}}
\end{array}\right],
$$

where $k_{\mu}$ and $k_{\sigma}$ are the kernel functions with respect to mean and standard deviation of the lognormal distributed variable $x$, respectively.

If $x$ is two parameter Weibull distributed variable, its probability density function can be written as follows:

$$
f(x)=\frac{\beta}{\alpha}\left(\frac{x}{\alpha}\right)^{\beta-1} e^{-(x / \alpha)^{\beta}},
$$

where $\beta$ is the shape parameter, and $\alpha$ is the scale parameter.

The kernel function of the Weibull distributed variable $X$ with respect to scale parameter $\alpha$ and shape parameter $\beta$ can be derived from equation (12) directly, which can be written as follows:

$$
\begin{aligned}
& k_{\alpha}=\frac{\beta}{\alpha}\left[\left(\frac{x}{\alpha}\right)^{\beta}-1\right], \\
& k_{\beta}=\frac{1}{\beta}+\left[1-\left(\frac{x}{\beta}\right)^{\beta}\right] \ln \left(\frac{x}{\alpha}\right) .
\end{aligned}
$$

The mean and standard deviation $\mu$ and $\sigma$ of $X$ can be written as follows:

$$
\begin{aligned}
& \mu=\alpha \cdot \Gamma\left(\frac{1}{\beta}+1\right), \\
& \sigma=\alpha \cdot \sqrt{\Gamma\left(\frac{2}{\beta}+1\right)-\left(\Gamma\left(\frac{1}{\beta}+1\right)\right)^{2}},
\end{aligned}
$$

where $\mu$ and $\sigma$ are the mean and standard deviation of $X$, respectively, and $\Gamma(\cdot)$ is the gamma function.

Calculate partial derivative of $\mu$ and $\sigma$ with respect to $\alpha$ and $\beta$, respectively, from equation (21); then,

$$
\begin{aligned}
& \frac{\partial \mu}{\partial \alpha}=\Gamma\left(\frac{1}{\beta}+1\right), \\
& \frac{\partial \sigma}{\partial \alpha}=\frac{\sigma}{\alpha}, \\
& \frac{\partial \mu}{\partial \beta}=-\frac{\alpha \Gamma((1 / \beta)+1) \Psi((1 / \beta)+1)}{\beta^{2}},
\end{aligned}
$$

$\frac{\partial \sigma}{\partial \beta}=\frac{\alpha^{2}\left[\Gamma^{2}((1 / \beta)+1) \Psi((1 / \beta)+1)-\Gamma((2 / \beta)+1) \Psi((2 / \beta)+1)\right]}{\beta^{2} \sigma}$,

where $\Psi(\cdot)$ is the psi function.

Jacobi matrix $\mathbf{J}$ can be written as follows: $\mathbf{J}=$ $\left[\begin{array}{ll}\partial \mu / \partial \alpha & \partial \sigma / \partial \alpha \\ \partial \mu / \partial \beta & \partial \sigma / \partial \beta\end{array}\right]$; then, the inverse matrix of $\mathbf{J}$ can be written as $\mathbf{J}^{-1}=\left[\begin{array}{ll}\partial \alpha / \partial \mu & \partial \beta / \partial \mu \\ \partial \alpha / \partial \sigma & \partial \beta / \partial \sigma\end{array}\right]$

The kernel function of the Weibull distributed variable $X$ with respect to its mean and standard deviation can be written as follows:

$$
\left[\begin{array}{c}
k_{\mu} \\
k_{\sigma}
\end{array}\right]=\left[\begin{array}{l}
\frac{\partial \alpha}{\partial \mu} k_{\alpha}+\frac{\partial \beta}{\partial \mu} k_{\beta} \\
\frac{\partial \alpha}{\partial \sigma} k_{\alpha}+\frac{\partial \beta}{\partial \sigma} k_{\beta}
\end{array}\right]=\left[\begin{array}{ll}
\frac{\partial \alpha}{\partial \mu} & \frac{\partial \beta}{\partial \mu} \\
\frac{\partial \alpha}{\partial \sigma} & \frac{\partial \beta}{\partial \sigma}
\end{array}\right] \cdot\left[\begin{array}{l}
k_{\alpha} \\
k_{\beta}
\end{array}\right]=\mathbf{J}^{-1} \cdot\left[\begin{array}{l}
k_{\alpha} \\
k_{\beta}
\end{array}\right],
$$

where $k_{\alpha}$ and $k_{\beta}$ are the kernel functions with respect to scale parameter and shape parameter, respectively. $\alpha$ and $\beta$ are the scale parameter and shape parameter of the two parameter Weibull distributed $X$, respectively.

\section{Reliability and Reliability Sensitivity Based on Edgeworth Series}

3.1. Reliability Based on Edgeworth Series. The failure probability of a mechanical component or a random structure can be calculated out by the following multidimensional integral:

$$
P_{\mathrm{f}}=P[g(\mathbf{X})<0]=\int_{g(\mathbf{X})<0} f_{\mathbf{X}}(\mathbf{x}) \mathrm{d} \mathbf{x},
$$

where $P_{f}$ is the failure probability of a random structure, $\mathbf{X}=\left[X_{1}, X_{2}, \Lambda, X_{N}\right]^{T} \in R^{N}$ represents the $N$-dimensional random input variables of the random structure, $f_{\mathbf{X}}(\mathbf{x})$ is the joint probability density function of random input variable $\mathbf{X}, g(\mathbf{X})$ is the performance function, $g(\mathbf{X})<0$ represents the failure domain, $g(\mathbf{X})>0$ represents the safety domain, and $g(\mathbf{X})=0$ represents the limit state. 
In practice, it is difficult to obtain the analytical solution of the multidimensional integral in equation (25) because of the nonlinear integration boundary $g(\mathbf{X})=0$ and high dimension of $\mathbf{X}$. There are two classes of approaches available for estimating the failure probability $P_{f}$ in equation (25), the analytical method and numerical simulation method. The numerical simulation method is the Monte Carlo simulation as known. Analytical methods (e.g., first-order reliability method (FORM)/second-order reliability method (SORM)), higher-order moment method, and saddlepoint approximation) can always be applied to calculate failure probability $P_{f}$ because of high computational efficiency and accuracy of them.

Suppose the first four central moments of performance function $g(\mathbf{X})$ are $\mu_{g}, v_{g}, \theta_{g}$, and $\eta_{g}$. The failure probability $P_{f}$ in equation (27) can be approximately estimated by Edgeworth series which can be written as follows:

$$
P_{f}(\zeta)=\Phi(\zeta)-\phi(\zeta)\left[\frac{1}{6} \frac{\theta_{g}}{\sigma_{g}^{3}} H_{2}(\zeta)+\frac{1}{24}\left(\frac{\eta_{g}}{\sigma_{g}^{4}}-3\right) H_{3}(\zeta)+\frac{1}{72}\left(\frac{\theta_{g}}{\sigma_{g}^{3}}\right)^{2} H_{5}(\zeta)\right]
$$

where $\Phi(\cdot)$ is the standard normal distribution function, $\varphi(\cdot)$ is the standard normal probability density, $\mu_{g}, v_{g}, \theta_{g}$, and $\eta_{g}$ are the mean, deviation, the third central moment, and the fourth central moment of performance function $g(\mathbf{X}), \sigma_{g}$ is the standard deviation of $g(\mathbf{X}), \varsigma=-\mu_{g} / \sigma_{h}$ is the negative reliability index, and $H_{j}(\cdot)$ is the $j^{\text {th }}$ Hermite polynomial and can be written as follows:

$$
\begin{aligned}
H_{j+1}(\zeta) & =2 \zeta H_{j}(\zeta)-2 j H_{j-1}(\zeta), \\
H_{0}(\zeta) & =1, \\
H_{1}(\zeta) & =2 \zeta .
\end{aligned}
$$

3.2. Reliability Sensitivity Based on Edgeworth Series. Partial derivative of failure probability $P_{f}$ in equation (28) with respect to the first four central moments $\mu_{g}, v_{g}, \theta_{g}$, and $\eta_{g}$ of performance function $g(\mathbf{X})$ can be written as follows:

$$
\begin{aligned}
& \frac{\partial P_{f}}{\partial \mu_{g}}=\frac{\partial P_{f}}{\partial \zeta} \frac{\partial \zeta}{\partial \mu_{g}} \\
& \frac{\partial P_{f}}{\partial \nu_{g}}=\left(\frac{\partial P_{f}}{\partial \zeta} \frac{\partial \zeta}{\partial \sigma_{g}}+\frac{\partial P_{f}}{\partial \sigma_{g}}\right) \frac{\partial \sigma_{g}}{\partial \nu_{g}}, \\
& \frac{\partial P_{f}}{\partial \theta_{g}}=-\phi(\zeta)\left[\frac{1}{6 \cdot \sigma_{g}^{3}} H_{2}(\zeta)+\frac{1}{36} \frac{\theta_{g}}{\sigma_{g}^{6}} H_{5}(\zeta)\right] \\
& \frac{\partial P_{f}}{\partial \eta_{g}}=-\phi(\zeta)\left[\frac{1}{24} \frac{\eta_{g}}{\sigma_{g}^{4}} H_{3}(\zeta)\right],
\end{aligned}
$$

where

$$
\begin{aligned}
& \frac{\partial P_{f}}{\partial \zeta}=\phi(\zeta)+\left\{\begin{array}{r}
\zeta \phi(\zeta)\left[\frac{1}{6} \frac{\theta_{g}}{\sigma_{g}^{3}} H_{2}(\zeta)+\frac{1}{24}\left(\frac{\eta_{g}}{\sigma_{g}^{4}}-3\right) H_{3}(\zeta)+\frac{1}{72}\left(\frac{\theta_{g}}{\sigma_{g}^{3}}\right)^{2} H_{5}(\zeta)\right]- \\
\phi(\zeta)\left[\frac{1}{3} \frac{\theta_{g}}{\sigma_{g}^{3}} H_{1}(\zeta)+\frac{1}{8}\left(\frac{\eta_{g}}{\sigma_{g}^{4}}-3\right) H_{2}(\zeta)+\frac{5}{72}\left(\frac{\theta_{g}}{\sigma_{g}^{3}}\right)^{2} H_{4}(\zeta)\right]
\end{array}\right\}, \\
& \frac{\partial \zeta}{\partial \mu_{g}}=-\frac{1}{\sigma_{g}}, \\
& \frac{\partial \zeta}{\partial \sigma_{g}}=\frac{\mu_{g}}{\sigma_{g}^{2}}, \\
& \frac{\partial \sigma_{g}}{\partial \nu_{g}}=\frac{1}{2 \sigma_{g}}, \\
& \frac{\partial P_{f}}{\partial \sigma_{g}}=\phi(\zeta)
\end{aligned}
$$


The first four central moments $\mu_{g}, v_{g}, \theta_{g}$, and $\eta_{g}$ of performance function $g(\mathbf{X})$ can be written as follows:

$$
\left\{\begin{array}{l}
\mu_{g}=m_{1}, \\
v_{g}=m_{2}-m_{1}^{2}, \\
\theta_{g}=m_{3}-3 m_{1} m_{2}+2 m_{1}^{3}, \\
\eta_{g}=m_{4}-4 m_{1} m_{3}+6 m_{1}^{2} m_{2}-3 m_{1}^{4},
\end{array}\right.
$$

where $m_{l}(l=1,2,3,4)$ are the first four origin moments of $g(\mathbf{X})=0$ which can be estimated by equation (7).

From equation (34), the partial derivative of central moments $\mu_{g}, v_{g}, \theta_{g}$, and $\eta_{g}$ of performance function $g(\mathbf{X})$ with respect to the distribution parameters $T_{j}^{i}(j=1,2, \ldots, N, i=1,2) \quad(i=1$ represents mean; $i=2$ represents standard deviation) of random inputs $\mathbf{X}=\left[X_{1}, X_{2}, \ldots, X_{N}\right]^{T}$ can be written as follows:

$$
\left\{\begin{array}{l}
\frac{\partial \mu_{g}}{\partial T_{j}^{i}}=\frac{\partial m_{1}}{\partial T_{j}^{i}}, \\
\frac{\partial \nu_{g}}{\partial T_{j}^{i}}=\frac{\partial m_{2}}{\partial T_{j}^{i}}-2 m_{1} \frac{\partial m_{1}}{\partial T_{j}^{i}}, \\
\frac{\partial \theta_{g}}{\partial T_{j}^{i}}=\frac{\partial m_{3}}{\partial T_{j}^{i}}-3\left(\frac{\partial m_{1}}{\partial T_{j}^{i}} m_{2}+\frac{\partial m_{2}}{\partial T_{j}^{i}} m_{1}\right)+6 m_{1}^{2} \frac{\partial m_{2}}{\partial T_{j}^{i}}, \\
\frac{\partial \eta_{g}}{\partial T_{j}^{i}}=\frac{\partial m_{4}}{\partial T_{j}^{i}}-4\left(\frac{\partial m_{1}}{\partial T_{j}^{i}} m_{3}+\frac{\partial m_{3}}{\partial T_{j}^{i}} m_{1}\right)+6\left(2 m_{1} m_{2} \frac{\partial m_{1}}{\partial T_{j}^{i}}+m_{1}^{2} \frac{\partial m_{2}}{\partial T_{j}^{i}}\right)-12 m_{1}^{3} \frac{\partial m_{1}}{\partial T_{j}^{i}},
\end{array}\right.
$$

$$
(i=1,2, j=1,2, \ldots, N) \text {, }
$$

where $\left(\partial m_{l} / \partial T_{j}^{i}\right)(l=1,2,3,4)$ can be calculated out by equation (11).

The failure probability sensitivity defined as partial derivative of failure probability $P_{f}$ with respect to distribution parameters $T_{j}^{i}$ of random inputs $\mathbf{X}=\left[X_{1}, X_{2}, \ldots, X_{N}\right]^{T}$ can be written as follows:

$$
\frac{\partial P_{f}}{\partial T_{j}^{i}}=\frac{\partial P_{f}}{\partial \mu_{g}} \frac{\partial \mu_{g}}{\partial T_{j}^{i}}+\frac{\partial P_{f}}{\partial \nu_{g}} \frac{\partial \nu_{g}}{\partial T_{j}^{i}}+\frac{\partial P_{f}}{\partial \theta_{g}} \frac{\partial \theta_{g}}{\partial T_{j}^{i}}+\frac{\partial P_{f}}{\partial \eta_{g}} \frac{\partial \eta_{g}}{\partial T_{j}^{i}}, \quad(j=1,2, \ldots, N, i=1,2,) .
$$

From equation (36), $\partial P_{j} / \partial T_{j}^{i}$ can be calculated out according to equations (28)-(31) and equation (35). The flow process diagram of the computational procedure is shown in Figure 1.

\section{Numerical Examples}

4.1. Example 1: Nonlinear Performance Function with Normal Variables. This example considers a nonlinear performance function which is the stress limit state function of a multileaf spring written as follows:

$$
g(X)=r-\frac{3 P l}{7 b h^{2}}
$$

where basic random variables $\mathbf{X}=[r, p, l, b, h]$ are the independent and identically distributed Gaussian random variables, $r$ is the material strength of the leaf spring, $p$ is the load, and $b, h$, and $l$ are the geometric dimension width, thickness, and span of the leaf spring, respectively. The mean and standard deviation of random variables $\mathbf{X}=[r, p, l, b, h]$ are listed in Table 2 [22].

The proposed method and MCS are applied to analyze the failure probability and reliability sensitivity, respectively, and their results are listed in Table 3 for comparison. The MCS results can be considered as accurate results and $N$ is the number of samples.

In Table 3, the results obtained by the proposed method have small errors compared with those obtained by MCS. Example 1 indicates that the proposed method is suitable for problems with nonlinear performance functions.

4.2. Example 2: Linear Performance Function with Six Lognormal Variables. This example considers a linear performance function, a plastic collapse mechanism of a one-bay frame which has been used as example 5 by Der et al. [28] and Zhao and Ono [29], written as follows: 


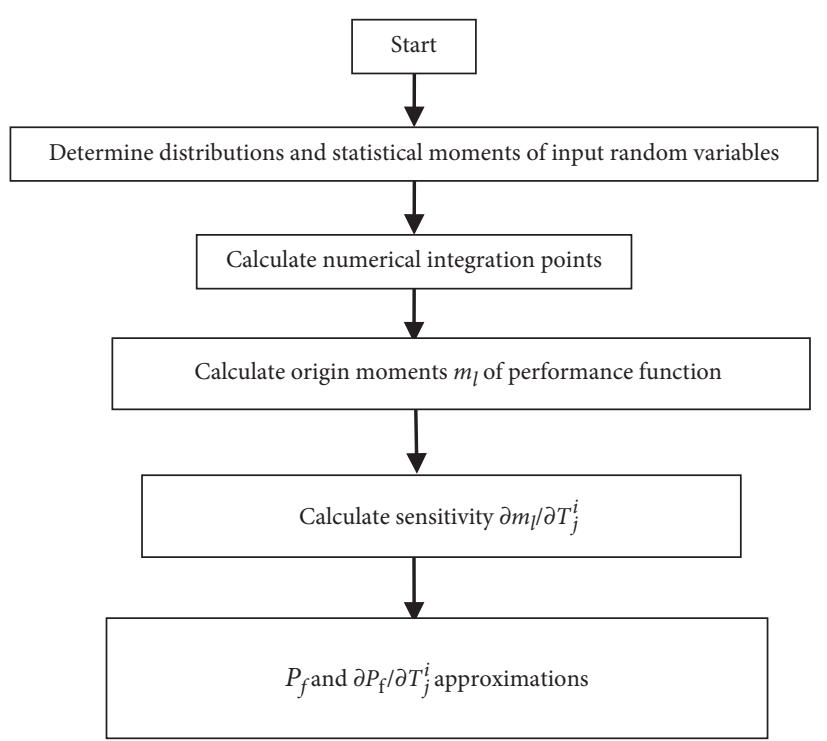

FIGURE 1: Flow process diagram for computations.

TABLE 2: Statistic moments and distributions of input variables of Example 1.

\begin{tabular}{lccccc}
\hline Distribution & Normal & Normal & Normal & Normal & Normal \\
\hline Variables & $r(\mathrm{MPa})$ & $P(\mathrm{~N})$ & $l(\mathrm{~mm})$ & $b(\mathrm{~mm})$ & 70 \\
Mean & 614 & 5537 & 1100 & 6.5 \\
Standard deviation & 45.8 & 276.85 & 5.5 & 0.35 & 0.0325 \\
\hline
\end{tabular}

TABLE 3: Failure probability and sensitivity results of Example 1.

\begin{tabular}{lcc}
\hline & Proposed method & MCS $(N=5 \times 106)$ \\
\hline$P_{\mathrm{f}}$ & $3.6904 \times 10^{-4}$ & $3.7501 \times 10^{-4}$ \\
$\partial P_{\mathrm{f}} / \partial \mu_{r}$ & $-2.6160 \times 10^{-5}$ & $-2.6826 \times 10^{-5}$ \\
$\partial P_{\mathrm{f}} / \partial \sigma_{r}$ & $7.9061 \times 10^{-5}$ & $7.8365 \times 10^{-5}$ \\
$\partial P_{\mathrm{f}} / \partial \mu_{h}$ & $-3.5000 \times 10^{-3}$ & $-3.9000 \times 10^{-3}$ \\
$\partial P_{\mathrm{f}} / \partial \sigma_{h}$ & $1.1000 \times 10^{-3}$ & $9.4906 \times 10^{-4}$ \\
$\partial P_{\mathrm{f}} / \partial \mu_{b}$ & $-1.6736 \times 10^{-4}$ & $-1.5067 \times 10^{-4}$ \\
$\partial P_{\mathrm{f}} / \partial \sigma_{b}$ & $2.5960 \times 10^{-5}$ & $2.7601 \times 10^{-5}$ \\
$\partial P_{\mathrm{f}} / \partial \mu_{l}$ & $1.0535 \times 10^{-5}$ & $1.1517 \times 10^{-5}$ \\
$\partial P_{\mathrm{f}} / \partial \sigma_{l}$ & $1.5250 \times 10^{-6}$ & $1.8140 \times 10^{-6}$ \\
$\partial P_{\mathrm{f}} / \partial \mu_{P}$ & $2.0798 \times 10^{-6}$ & $2.1164 \times 10^{-6}$ \\
$\partial P_{\mathrm{f}} / \partial \sigma_{P}$ & $3.0267 \times 10^{-6}$ & $3.0777 \times 10^{-6}$ \\
\hline
\end{tabular}

$$
g(X)=x_{1}+2 x_{2}+2 x_{3}+x_{4}-5 x_{5}-5 x_{6},
$$

where basic random variables $\mathbf{X}=\left[x_{1}, x_{2}, x_{3}, x_{4}, x_{5}, x_{6}\right]$ are the independent and identically distributed lognormal random variables. The mean and standard deviation of random variables $\mathbf{X}=\left[x_{1}, x_{2}, x_{3}, x_{4}, x_{5}, x_{6}\right]$ are listed in Table 4.

The proposed method and MCS are applied to analyze the failure probability and reliability sensitivity, respectively, and their results are listed in Table 5 for comparison.

In Table 5, the results obtained by the proposed method have small errors compared with those obtained by MCS. Example 2 indicates that the proposed method is suitable for problems with nonnormal random input variables.
4.3. Example 3: Nonlinear Performance Function with Nonnormal Variables. This example considers a nonlinear performance function which describes the displacement response of a $I$ section cantilever beam shown in Figure 2 written as follows:

$$
g(X)=d-\frac{4 F l^{3}}{E\left[b h^{3}-b(h-2 t)^{3}+a(h-2 t)^{3}\right]},
$$

where basic random variables $\mathbf{X}=[a, t, b, h, l, F, d, E]$ are the independent random variables. Here, $a$ is the thickness of web, $t$ is the thickness of flange, $b$ is the width of $I$-beam section, $h$ is the height of $I$-beam section, $l$ is the length of cantilever beam, $f$ is the load force, $d$ is the allowable maximum deformation, and $E$ is the elastic modulus. The 
TABLE 4: Statistic moments and distributions of input variables of Example 2.

\begin{tabular}{lcccccc}
\hline Distribution & Lognormal & Lognormal & Lognormal & Lognormal & Lognormal & Lognormal \\
\hline Variables & $x_{1}$ & $x_{2}$ & $x_{3}$ & $x_{4}$ & $x_{5}$ & $x_{6}$ \\
Mean & 120 & 120 & 120 & 120 & 50 & 40 \\
Standard deviation & 12 & 12 & 12 & 12 & 15 & 12 \\
\hline
\end{tabular}

TABLE 5: Failure probability and sensitivity results of Example 2.

\begin{tabular}{lcc}
\hline & Proposed method & MCS $\left(N=5 \times 10^{6}\right)$ \\
\hline$P_{\mathrm{f}}$ & $1.3600 \times 10^{-2}$ & $1.204 \times 10^{-2}$ \\
$\partial P_{\mathrm{f}} / \partial \mu_{1}$ & $-2.2290 \times 10^{-4}$ & $-1.942 \times 10^{-4}$ \\
$\partial P_{\mathrm{f}} / \partial \sigma_{1}$ & $3.4596 \times 10^{-5}$ & $4.104 \times 10^{-5}$ \\
$\partial P_{\mathrm{f}} / \partial \mu_{2}$ & $-4.4554 \times 10^{-4}$ & $-4.068 \times 10^{-4}$ \\
$\partial P_{\mathrm{f}} / \partial \sigma_{2}$ & $1.3341 \times 10^{-4}$ & $1.610 \times 10^{-4}$ \\
$\partial P_{\mathrm{f}} / \partial \mu_{3}$ & $-4.4554 \times 10^{-4}$ & $-4.072 \times 10^{-4}$ \\
$\partial P_{\mathrm{f}} / \partial \sigma_{3}$ & $1.3341 \times 10^{-4}$ & $1.429 \times 10^{-4}$ \\
$\partial P_{\mathrm{f}} / \partial \mu_{4}$ & $-2.2290 \times 10^{-4}$ & $-2.342 \times 10^{-4}$ \\
$\partial P_{\mathrm{f}} / \partial \sigma_{4}$ & $3.4596 \times 10^{-5}$ & $7.114 \times 10^{-5}$ \\
$\partial P_{\mathrm{f}} / \partial \mu_{5}$ & $9.5913 \times 10^{-4}$ & $9.157 \times 10^{-4}$ \\
$\partial P_{\mathrm{f}} / \partial \sigma_{5}$ & $2.9000 \times 10^{-3}$ & $2.482 \times 10^{-3}$ \\
$\partial P_{\mathrm{f}} / \partial \mu_{6}$ & $1.0000 \times 10^{-3}$ & $9.500 \times 10^{-4}$ \\
$\partial P_{\mathrm{f}} / \partial \sigma_{6}$ & $1.9000 \times 10^{-3}$ & $1.732 \times 10^{-3}$ \\
\hline
\end{tabular}

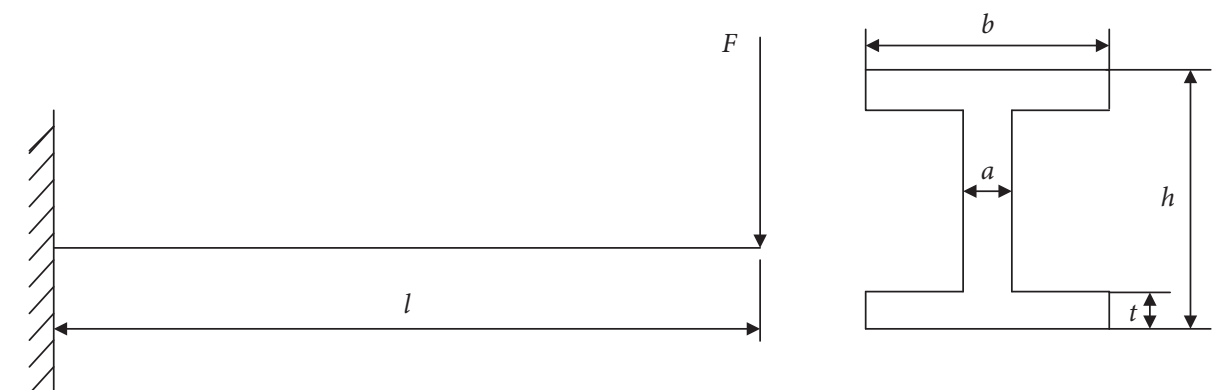

FIGURE 2: Section cantilever beam.

TABLE 6: Statistic moments and distributions of input variables of Example 2.

\begin{tabular}{lcccccccc}
\hline Distribution & Normal & Normal & Normal & Normal & Normal & Weibull & Lognormal & Lognormal \\
\hline Variables & $a(\mathrm{~mm})$ & $t(\mathrm{~mm})$ & $b(\mathrm{~mm})$ & $h(\mathrm{~mm})$ & $l(\mathrm{~mm})$ & $F(\mathrm{~N})$ & $d(\mathrm{~mm})$ & $E(\mathrm{MPa})$ \\
Mean & 10 & 14 & 88 & 205 & 2000 & $5.187 \times 10^{4}$ & 27 & $2.03 \times 10^{5}$ \\
Standard deviation & 0.05 & 0.07 & 0.44 & 1.025 & 10 & 213.865 & 0.135 & $1.015 \times 10^{3}$ \\
\hline
\end{tabular}

distributions and distribution parameters (mean and standard deviation) of these input random variables are listed in Table 6.

The scale parameter and shape parameter of random variable $F$ are $\alpha=5.197 \times 10^{4}$ and $\beta=310.36$, respectively.

The proposed method and MCS are applied to analyze the failure probability and reliability sensitivity, respectively, and their results are listed in Table 7 for comparison.
In Table 7, the results obtained by the proposed method have small errors compared with those obtained by MCS. Example 3 indicates that the proposed method is suitable for problems with nonlinear performance functions with nonnormal random input variables. The nonnormal variables need not to be transformed into equivalent normal ones. But the coefficient of variation of the input random variables should be small ( $\leq 0.1$ for Weibull distribution variables). 
TABLE 7: Failure probability and sensitivity results of Example 3.

\begin{tabular}{lcc}
\hline & Proposed method & MCS $\left(N=5 \times 10^{6}\right)$ \\
\hline$P_{\mathrm{f}}$ & $9.7363 \times 10^{-4}$ & $2.8000 \times 10^{-4}$ \\
$\partial P_{\mathrm{f}} / \partial \mu_{a}$ & $-8.2470 \times 10^{-4}$ & $-5.0299 \times 10^{-4}$ \\
$\partial P_{\mathrm{f}} / \partial \sigma_{a}$ & $1.0579 \times 10^{-4}$ & $1.1908 \times 10^{-4}$ \\
$\partial P_{\mathrm{f}} / \partial \mu_{t}$ & $-2.2000 \times 10^{-3}$ & $-2.0500 \times 10^{-3}$ \\
$\partial P_{\mathrm{f}} / \partial \sigma_{t}$ & $1.1000 \times 10^{-3}$ & $1.4000 \times 10^{-3}$ \\
$\partial P_{\mathrm{f}} / \partial \mu_{b}$ & $-4.4905 \times 10^{-4}$ & $-5.8711 \times 10^{-4}$ \\
$\partial P_{\mathrm{f}} / \partial \sigma_{b}$ & $3.2129 \times 10^{-4}$ & $1.8541 \times 10^{-4}$ \\
$\partial P_{\mathrm{f}} / \partial \mu_{h}$ & $-5.5812 \times 10^{-4}$ & $-6.4358 \times 10^{-4}$ \\
$\partial P_{\mathrm{f}} / \partial \sigma_{h}$ & $1.1000 \times 10^{-3}$ & $1.5000 \times 10^{-3}$ \\
$\partial P_{\mathrm{f}} / \partial \mu_{l}$ & $9.3914 \times 10^{-5}$ & $8.9505 \times 10^{-5}$ \\
$\partial P_{\mathrm{f}} / \partial \sigma_{l}$ & $1.9045 \times 10^{-4}$ & $1.9436 \times 10^{-4}$ \\
$\partial P_{\mathrm{f}} / \partial \mu_{F}$ & $5.5696 \times 10^{-6}$ & $1.5504 \times 10^{-6}$ \\
$\partial P_{\mathrm{f}} / \partial \sigma_{F}$ & $8.4887 \times 10^{-5}$ & $8.8620 \times 10^{-7}$ \\
$\partial P_{\mathrm{f}} / \partial \mu_{d}$ & $-1.9000 \times 10^{-3}$ & $-2.1000 \times 10^{-3}$ \\
$\partial P_{\mathrm{f}} / \partial \sigma_{d}$ & $1.6000 \times 10^{-3}$ & $1.5000 \times 10^{-3}$ \\
$\partial P_{\mathrm{f}} / \partial \mu_{E}$ & $-2.2514 \times 10^{-7}$ & $-3.6546 \times 10^{-7}$ \\
$\partial P_{\mathrm{f}} / \partial \sigma_{E}$ & $1.8949 \times 10^{-7}$ & $2.5002 \times 10^{-7}$ \\
\hline
\end{tabular}

\section{Conclusions}

The univariate dimension-reduction method, reliability analysis based on Edgeworth series, and reliability sensitivity analysis are employed to present a computational procedure for estimating failure probability sensitivity of random engineering structures. Three numerical examples are employed to illustrate the performance of the proposed method. To illustrate the feasibility of the proposed method, failure probability and failure probability sensitivity results obtained by the new method are compared with those obtained by direct MCS. It is concluded as follows: The proposed method is suitable for models of which the input random variables have small coefficient of variation $(\leq 0.1)$. The nonnormal input variables need not to be transformed into equivalent normal ones, so the new method can be conveniently applied to solve problems with nonnormal input variables.

\section{Data Availability}

The data and models used to support the findings of the study are included within the article.

\section{Conflicts of Interest}

The authors declare that they have no conflicts of interest.

\section{Acknowledgments}

This study was supported by the Self-Financing Project of Scientific Research and Development Plan of LangFang Science and Technology Bureau (2020011022) and the Fundamental Research Funds for the Central Universities of China (2020011022 and ZY20215140).

\section{References}

[1] J. Wu, D. Zhang, J. Liu, X. Jia, and X. Han, “A computational framework of kinematic accuracy reliability analysis for industrial robots," Applied Mathematical Modelling, vol. 82, no. 82, pp. 189-216, 2020.

[2] J. Wu, D. Zhang, C. Jiang et al., "On reliability analysis method through rotational sparse grid nodes," Mechanical Systems and Signal Processing, vol. 147, Article ID 107106, 2021.

[3] D. Meng, T. Xie, P. Wu, C. He, Z. Hu, and Z. Lv, "An uncertainty-based design optimization strategy with random and interval variables for multidisciplinary engineering systems," Structures, vol. 32, no. 32, pp. 997-1004, 2021.

[4] D. Meng, T. Xie, P. Wu, S.-P. Zhu, Z. Hu, and Y. Li, "Uncertainty-based design and optimization using first order saddlepoint approximation method for multidisciplinary engineering systems," ASCE-ASME Journal of Risk and Uncertainty in Engineering Systems, Part A: Civil Engineering, vol. 6, no. 3, Article ID 104020028, 2020.

[5] R. E. Melchers and M. Ahammed, "A fast approximate method for parameter sensitivity estimation in Monte Carlo structural reliability," Computers \& Structures, vol. 82, no. 1, pp. 55-61, 2004.

[6] Y. T. Wu and S. Mohanty, "Variable screening and ranking using sampling-based sensitivity measures," Reliability Engineering \& System Safety, vol. 91, no. 6, pp. 634-647, 2006.

[7] Y. Wu, "Computational methods for efficient structural reliability and reliability sensitivity analysis," AIAA Joural, vol. 32, no. 8, pp. 1717-1723, 1994.

[8] M. Ahammed and R. E. Melchers, "Gradient and parameter sensitivity estimation for systems evaluated using Monte Carlo analysis," Reliability Engineering \& System Safety, vol. 91, no. 5, pp. 594-601, 2006.

[9] S. K. Au, "Reliability-based design sensitivity by efficient simulation," Computers \& Structures, vol. 83, no. 14, pp. 1048-1061, 2005. 
[10] Z. Z. Lu, S. F. Song, Z. F. Yue et al., "Reliability sensitivity method by line sampling," Structural Safety, vol. 30, no. 6, pp. 517-532, 2008.

[11] W. Zhao, Y. Chen, and J. Liu, "An effective first order reliability method based on Barzilai-Borwein step," Applied Mathematical Modelling, vol. 77, no. Pt 2, pp. 1545-1563, 2020.

[12] A. J. Torii, R. H. Lopez, and L. Miguel, "A second order SAP algorithm for risk and reliability based design optimization," Reliability Engineering \& System Safety, vol. 190, pp. 1-11, Article ID 106499, 2019.

[13] W. Fan, "Adaptive anisotropic response surface method based on univariate dimension-reduction model and its high-order revision," Engineering Computations, vol. 37, no. 9, 3125 pages, 2020.

[14] M. Hohenbichler and R. Rackwitz, "Sensitivity and importance measures in structural reliability," Civil Engineering Systems, vol. 3, no. 2, pp. 203-209, 1986.

[15] P. Bjerager and S. Krenk, "Parametric sensitivity in first order reliability theory," Journal of Engineering Mechanics, vol. 115, no. 7, pp. 1577-1582, 1989.

[16] A. Karamchandani and C. A. Cornell, "Sensitivity estimation within first and second order reliability methods," Structural Safety, vol. 11, no. 2, pp. 95-107, 1991.

[17] Y. M. Zhang, X. D. He, Q. L. Liu et al., "Reliability sensitivity of automobile components with arbitrary distribution parameters," Proceedings of the Institution of Mechanical Engineers Part D-Journal of Automobile Engineering, vol. 219, no. D2, pp. 165-182, 2005.

[18] R. H. Sues and M. A. Cesare, "System reliability and sensitivity factors via the MPPSS method," Probability Engineering Mechanics, vol. 20, pp. 148-157, 2005.

[19] Z. Lu, J. Song, S. Song, Z. Yue, and J. Wang, "Reliability sensitivity by method of moments," Applied Mathematical Modelling, vol. 34, no. 10, pp. 2860-2871, 2010.

[20] D. Meng, Z. Hu, P. Wu et al., "Reliability-based optimization for offshore structures using saddlepoint approximation," Proceedings of the Institution of Civil Engineers-Maritime Engineering, vol. 173, no. 2, pp. 33-42, 2020.

[21] D. Meng, Y. Li, C. He, J. Guo, Z. Lv, and P. Wu, "Multidisciplinary design for structural integrity using a collaborative optimization method based on adaptive surrogate modelling," Materials \& Design, vol. 206, Article ID 109789, 2021.

[22] Y. L. Zhang and Y. M. Zhang, "A new stochastic analysis method for mechanical components," Proceedings of the Institution of Mechanical Engineers, Part C: Journal of Mechanical Engineering Science, vol. 227, no. 8, pp. 1818-1829, 2013.

[23] Li-sha Zhu, L.-sheng Li, S.-jie Li, and S. Wang, "Research on reliability for servo turret based on vibration transmission path system with stiffness degradation model," Mathematical Problems in Engineering, vol. 2020, no. 3, 11 pages, Article ID 3764546, 2020.

[24] S. Rahman and H. Xu, "A univariate dimension-reduction method for multi-dimensional integration in stochastic mechanics," Probabilistic Engineering Mechanics, vol. 19, no. 4, pp. 393-408, 2004.

[25] H. Xu and S. Rahman, "Decomposition methods for structural reliability analysis," Probabilistic Engineering Mechanics, vol. 20, no. 3, pp. 239-250, 2005.

[26] H. Xu and S. Rahman, "A generalized dimension-reduction method for multi-dimensional integration in stochastic mechanics," International Journal for Numerical Methods in Engineering, vol. 65, no. 13, pp. 1992-2019, 2006.

[27] X. F. Zhang, M. D. Pandey, and Y. M. Zhang, "A numerical method for structural uncertainty computation," Science China Technological Sciences, vol. 054, no. 012, pp. 3347-3357, 2011.

[28] K. A.. Der, H. Z. Lin, and S. J. Hwang, "Second-order reliability approximations," Journal of Engineering Mechanics ASCE, vol. 113, no. 8, pp. 1208-1225, 1987.

[29] Y. G. Zhao and T. Ono, "Moment methods for structural reliability," Structural Safety, vol. 23, no. 1, pp. 47-75, 2001. 